\title{
The Capacity of Visual Short-Term Memory is Set Both by Visual Information Load and by Number of Objects
}

\section{Citation}

Alvarez, G., and P. Cavanagh. 2004. "The capacity of visual short-term memory is set both by visual information load and by number of objects.." Psychological Science 15 (2): 106-11. DOI: 10.1111/j.0963-7214.2004.01502006.x

\section{Permanent link}

http://nrs.harvard.edu/urn-3:HUL.InstRepos:41302706

\section{Terms of Use}

This article was downloaded from Harvard University's DASH repository, WARNING: This file should NOT have been available for downloading from Harvard University's DASH repository.

\section{Share Your Story}

The Harvard community has made this article openly available.

Please share how this access benefits you. Submit a story.

\section{Accessibility}


Research Article

\title{
The Capacity of Visual Short- Term Memory Is Set Both by Visual Information Load and by Number of Objects
}

\author{
G.A. Alvarez and P. Cavanagh
}

Harvard University

\begin{abstract}
Previous research has suggested that visual shortterm memory has a fixed capacity of about four objects. However, we found that capacity varied substantially across the five stimulus classes we examined, ranging from 1.6 for shaded cubes to 4.4 for colors (estimated using a change detection task). We also estimated the information load per item in each class, using visual search rate. The changes we measured in memory capacity across classes were almost exactly mirrored by changes in the opposite direction in visual search rate $\left(\mathrm{r}^{2}=.992\right.$ between search rate and the reciprocal of memory capacity). The greater the information load of each item in a stimulus class (as indicated by a slower search rate), the fewer items from that class one can hold in memory. Extrapolating this linear relationship reveals that there is also an upper bound on capacity of approximately four or five objects. Thus, both the visual information load and number of objects impose capacity limits on visual short-term memory.
\end{abstract}

One of the most important components of any cognitive activity is the short-term storage and manipulation of information in memory. Many researchers assume that working memory can be divided into separate components for the storage of visual and verbal materials (Baddeley, $1978,1992)$. Moreover, within the visual modality, working memory can be divided into a high-capacity sensory memory and a relatively limited-capacity short-term memory (Phillips, 1974). Recent research (Luck \& Vogel, 1997; Vogel, Woodman, \& Luck, 2001) has shown that visual short-term memory appears to be limited by the number of objects that can be stored, independently of the number of features probed for each object. For example, it is possible to store one feature (e.g., color or orientation) of up to four objects. Surprisingly, it is also possible to remember two or even four features for up to approximately

Address correspondence to G.A. Alvarez, Department of Psychology, Harvard University, 33 Kirkland St., 7th Floor, Cambridge, MA 02138; e-mail: geoalvarez@wjh.harvard.edu. the same limit-four objects. On the basis of these results, Luck and Vogel claimed that the capacity of visual short-term memory is set in terms of the number of objects that can be stored, and not the number of features.

In this article, we reexamine whether the number of features is a factor in determining memory capacity. We address this alternative in terms of visual information. Specifically, we tested whether there is a fixed total amount of visual information that can be stored in visual short-term memory. We use the term "visual information" to refer to the visual features or details of an object that are encoded and stored in memory, and not to refer to information in the mathematical sense of classical information theory (Shannon, 1948). Thus, the visual information load of a particular object corresponds to the amount of visual detail stored for the object. The total visual information load is simply the sum of the visual information stored for each object in memory.

We tested whether visual short-term memory capacity is fixed in terms of the number of objects that can be stored or the total amount of visual information that can be stored. We took a behavioral measure, processing rate in a visual search task, to estimate the visual information load per object for five stimulus classes, colors, polygons, Chinese characters, shaded cubes, and letters (see Fig. 1). We assume that the more visual information that must be analyzed per object, the slower the processing rate. Although visual search rate is not a pure measure of the amount of detail encoded, it is reasonable to assume that visual search rate will increase monotonically with increases in the information content of objects, and it therefore serves as a useful index of the visual information load per object. A change detection task was used to estimate memory capacity in terms of the maximum number of objects that could be stored for the same five stimulus classes. The change detection task has been shown previously to yield highly similar performance with and without a verbal memory load (Luck \& Vogel, 1997). Thus, change detection provides relatively uncontaminated estimates of visual short-term memory capacity.

Of principal interest was whether there is a relationship between visual search rate and the inverse of memory capacity. If there is a limit to the total amount of visual information that can be stored in 


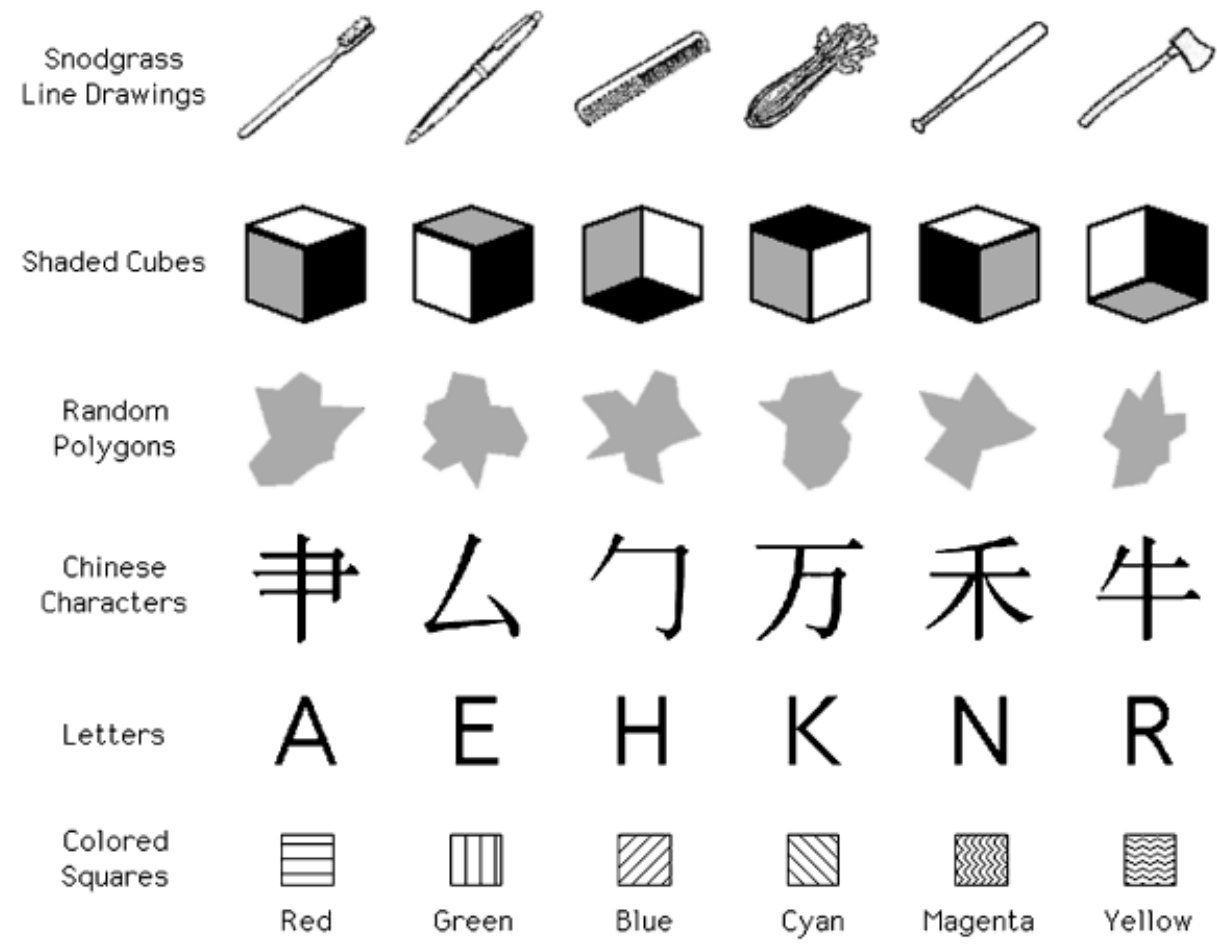

Fig. 1. The sets of objects used in the main experiment.

memory, there should be a monotonic relationship between search rate and the inverse of memory capacity. Specifically, as the information per item increases, the total number of items that add up to the fixed maximum of information will decrease. If that total information capacity is $C$ and the amount of information per item is $I$, then a maximum of $N$ items will fit in memory:

$$
I \times N=C
$$

and

$$
I=C / N
$$

The information per item estimated with visual search rate is then at least monotonically related to the reciprocal of the number of items that fit in visual short-term memory. Conversely, if the limiting factor in visual short-term memory is the number of objects that can be stored, then this number, $N$, should be constant regardless of the information load per object.

\section{METHOD}

\section{Participants}

The subjects included author G.A. and 5 naive subjects. Subjects ranged in age from 18 to 30 years, and each had normal or correctedto-normal visual acuity.

\section{Apparatus}

The experiments were run on an Apple Macintosh computer with custom software written in C using the Vision Shell Graphics Libraries (Comtois, 2003).
Stimuli

In the practice phase, six line drawings from the Snodgrass and Vanderwart (1980) set were used (images provided courtesy of Michael J. Tarr, Brown University, Providence, RI), and in the test phase, six objects from each of five different classes of stimuli were used (see Fig. 1). The objects subtended between $1.5^{\circ} \times 1.5^{\circ}$ and $3^{\circ} \times 3^{\circ}$ of visual angle (line drawings approximately $2.5^{\circ} \times 3^{\circ}$; shaded cubes, $3^{\circ} \times 3^{\circ}$; random polygons, $3^{\circ} \times 3^{\circ}$; Chinese characters, $2.5^{\circ} \times 2.5^{\circ}$; letters, $1.5^{\circ} \times 2^{\circ}$; colored squares, $1.5^{\circ} \times 1.5^{\circ}$ ). All stimuli were presented on a white background. The line drawings, Chinese characters, and letters were black. The random polygons were gray. The shaded cubes had a black outline frame with one white surface, one black surface, and one gray surface. The colored squares were red, green, blue, magenta, cyan, and yellow.

Stimuli were presented in pseudorandom positions within a $5 \times 4$ grid subtending $25^{\circ} \times 20^{\circ}$. Each item was randomly jittered $\pm 1^{\circ}$ horizontally and vertically from the center of the cell in which it was drawn.

\section{Procedure}

On each visual search trial, a target was presented at the center of the display for $500 \mathrm{~ms}$, followed by a 900 -ms blank interval, and then by the presentation of an array of 4,8 , or 12 objects from the same stimulus class as the target. The target was present in the array on half the trials, and subjects indicated as quickly as possible whether the target was present or absent. Visual search rate was estimated by taking the slope of the line relating target-present reaction time to the number of objects in the display. On each change detection trial, 1, 3, $5,7,9,11,13$, or 15 objects from one stimulus class were presented for $500 \mathrm{~ms}$, followed by a $900-\mathrm{ms}$ blank interval, and then by a second 
presentation of objects. On half of the trials, the two displays were identical, and on the other half, one of the objects changed identity. The subject's task was to indicate whether one of the objects changed. The number of objects yielding $75 \%$ correct performance on the change detection task was estimated for each stimulus class (see the appendix at http://www.blackwellpublishing.com/products/journals/ suppmat/alvarez/alvarez_appendix.html for a model of the relationship between the $75 \%$-correct threshold and capacity).

During the practice phase, each subject completed two sets of 90 trials of the visual search task and two sets of 144 trials of the change detection task with the line drawings. During the test phase, for each of the five classes of test stimuli, subjects completed two sets of 12 practice trials and 78 test trials on the visual search task and two sets of 16 practice trials and 128 test trials on the change detection task. The test phase was divided into 10 sessions, each consisting of one block of search trials and one block of change detection trials using the same stimulus class. The order of the search and change detection blocks alternated from one session to the next. Half of the subjects performed the 10 sessions in the following order: Chinese characters, letters, random polygons, cubes, colors, colors, cubes, random polygons, letters, and Chinese characters. For the other half of the subjects, the order of the stimulus classes was reversed (i.e., the sessions began with color).

\section{RESULTS}

For each individual subject, the upper and lower $2.5 \%$ of reaction times on the visual search task were trimmed from each condition to eliminate outliers. For the remaining trials, error rates on the search task varied across conditions, $F(4,20)=6.537, p<.01$ (colors: $4 \%$; letters: $4 \%$; Chinese characters: $7 \%$; polygons: $11 \%$; cubes: $13 \%$; all averaged across subjects). Overall, percentage error was low $(7 \% \pm 2 \%)$, and there was no evidence of a speed-accuracy trade-off. Visual search rate was estimated individually for each subject and each class of stimulus from the linear regression between the reaction time data of the target-present trials and the display size (these regressions typically accounted for more than $90 \%$ of the variance, indicating that the functions were reasonably linear). The visual search rates varied as a function of stimulus class, ranging from $11 \mathrm{~ms} / \mathrm{item}$ for colors to $127 \mathrm{~ms} /$ item for cubes (averaged across subjects), $F(4,20)=53.314$, $p<.01$.

In the change detection task, the percentage correct (averaged over change and no-change trials combined) was used to derive the $75 \%$ correct threshold number for each stimulus class individually for each subject. These thresholds were not constant in terms of the number of objects over the stimulus classes tested (ranging from 3.5 for cubes to 7.2 for colors, averaged across subjects), $F(4,20)=$ $6.026, p<.01$.

Figure 2 shows that visual search rate (averaged across subjects) is linearly related to the inverse of the $75 \%$ threshold number of objects, $r^{2}=.992, t(3)=19.28, p<.01$. This linear relationship was significant (at $p=.05)$ for 4 of the 6 subjects $(r=.94, .92, .98, .98 ; 2$ not significant: $r=.81, .17)$.

How do the $75 \%$-correct thresholds relate to memory capacity? If capacity is roughly estimated as half the $75 \%$-threshold number, the capacities range from 1.7 for cubes to 3.6 for colors, and the maximum capacity (where the linear function intersects the horizontal axis) is 4 . This simple model assumes that the observer answers correctly on

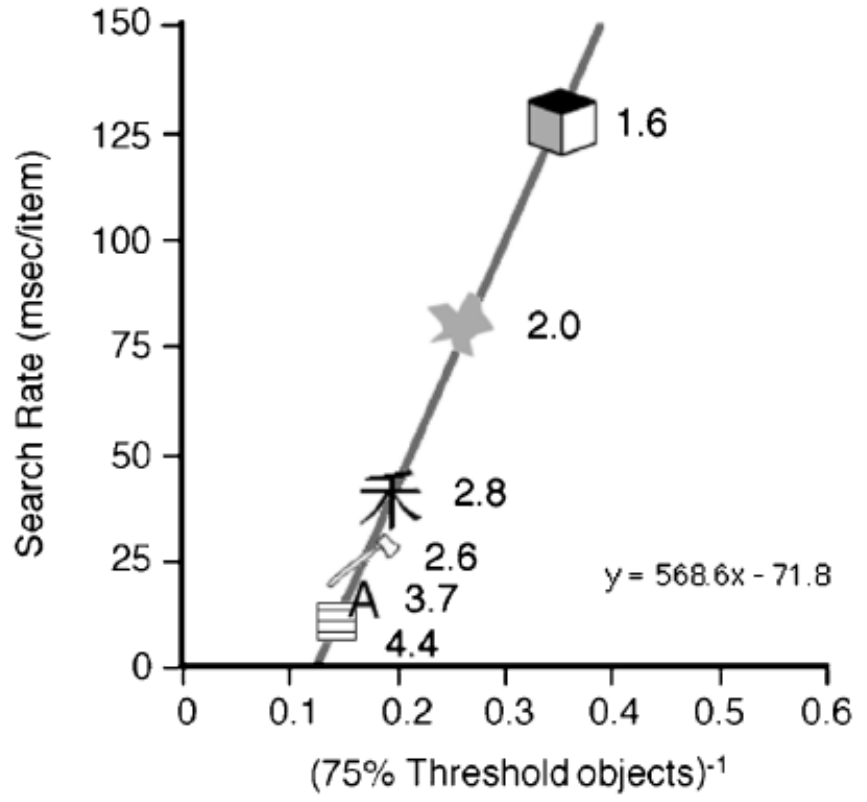

Fig. 2. Search rate as a function of the inverse of the $75 \%$-threshold number of objects in change detection for each stimulus class in the main experiment $\left(r^{2}=.992\right)$. Capacity estimates in terms of the number of objects (based on Pashler, 1988; see the appendix at http://www. blackwellpublishing.com/products/journals/suppmat/alvarez/alvarez_ appendix.html) are presented next to the symbols. Note that the graph includes a data point for line drawings, for which each subject completed the search task and change detection task first in a block of practice trials. The $r^{2}$ value is the same with or without this data point included.

change trials when the changed item is in memory and responds "no change" otherwise. A more reasonable model that takes hits and false alarms into account separately (see the appendix at http://www. blackwellpublishing.com/products/journals/suppmat/alvarez/alvarez_ appendix.html) generates very similar estimates ranging from 1.6 for cubes to 4.4 for colors, and a comparable upper bound of approximately 4.7 objects for the maximum capacity.

These data reveal that capacity, as estimated by the change detection task, is not constant across the range of visual materials tested. The greater the information load of each item in a stimulus class (as indicated by a slower search rate), the fewer objects from that class one can hold in memory. The strongly linear relation between information per item and the inverse of capacity suggests that capacity is limited by the total amount of information. Note that the linearity of the data is not essential for our claim of an information limit on visual short-term memory. That is, assuming there is an information limit, increases in visual search rate (corresponding to increases in the information per object) should always correspond to decreases in the number of objects stored, but the relationship does not have to be linear to show this trade-off.

It is also important to note that the observed relation is not exact, because the intercept of the regression line does not fall through the origin. This intercept falls at a $75 \%$ threshold of eight objects, equivalent to a capacity of four to five objects (see the appendix). This indicates that there is an upper limit to the number of objects that can be stored in visual short-term memory even if the individual items are so simple that they carry no information (at the theoretical lower 
bound on visual search rate of $0 \mathrm{~ms} /$ item). ${ }^{1}$ We address this point in more detail in the Discussion. Before doing so, it is necessary to first rule out three alternatives to the fixed information limit proposed to account for the linear relation between search rate and the inverse of memory capacity.

First, the visual search rate reflects, among other things, the time needed to encode each item in the display. A stimulus class with slow search rates would also require significant encoding time in the change detection task. Subjects were given only $500 \mathrm{~ms}$ to encode all the items in the change detection task, and it is possible that this was not enough time to encode all the items that could fit into memory. This encoding (as opposed to storage) limit would have led to an underestimate of capacity. To investigate this possibility, we ran a control experiment using only cubes, which had the slowest processing rate in the main experiment $(127 \mathrm{~ms} / \mathrm{item})$, and varying the presentation duration from $50 \mathrm{~ms}$ to a maximum of $850 \mathrm{~ms}$. Accuracy reached a maximum at $450 \mathrm{~ms}$ and did not improve for any longer presentations. Thus, insufficient presentation time for encoding the slowest stimuli cannot account for their lower capacity estimates in the main experiment. All the information that can be stored is acquired in less than $500 \mathrm{~ms}$, even for the objects that are processed at very slow rates.

A second alternative explanation for the covariation in search rate and change detection performance concerns the visual similarity of objects. If some errors in change detection occur during the comparison between objects in memory and the test display, then the number of such errors might increase as the similarity between the objects increases. To control for the role of visual similarity, we ran an additional control experiment in which we measured visual search rate and change detection performance for the same physical objects (block $2 \mathrm{~s}$ and $5 \mathrm{~s}$ ) rotated at several different angles between $0^{\circ}$ (ㄷ) and $90^{\circ}$ (ПபЛ). Six subjects (author G.A. and 5 new subjects) participated. The results replicated those of the main experiment, showing a strong linear relationship between search rate and the $75 \%$ threshold number of objects in change detection, $r^{2}=.81, t(4)=4.18$, $p<.05$, and a similar upper limit of approximately four objects in the intercept of the linear relation (when converted to capacity). Thus, the inverse relation between visual search rate and memory capacity holds even when the visual similarity of the objects is held constant.

Finally, we consider the alternative of a memory with a fixed number of slots, each holding one object and being limited in the number of features it can take. As the number of details per object increases for more complex objects, details in excess of this object limit are lost. According to this model, the maximum number of objects that can be stored is the same for all stimuli, but more complex objects are stored with lower precision, resulting in lower capacity estimates in the change detection task. However, this model must also predict poor performance even when a single complex object is stored (with reduced resolution) in memory. In contrast, our model follows standard capacity models in predicting good performance until the capacity limit is exceeded. We therefore predicted equivalent performance at set size 1 for all the stimulus classes we tested (which all had capacity estimates greater than 1). An analysis of change

${ }^{1}$ Note that a visual search rate of zero in the current experiment is only hypothetical. Given the type of search display used (heterogeneous displays with high target-distractor and distractor-distractor similarity), there is no stimulus type one would expect to yield a search slope of zero in this task. detection accuracy at set size 1 in the main experiment revealed that performance was high for each type of stimulus (ranging from $96 \%$ for cubes to $99 \%$ for polygons). There was no significant effect of stimulus type on change detection accuracy at this set size, $F(4,20)<1, p>.05$, and none of the pair-wise comparisons between conditions were significant (Fisher's $P L S D=4.25, p=.05$ ). Thus, we can reject the hypothesis that visual short-term memory consists of a fixed number of low-resolution slots.

Clearly, even our most complex stimuli were stored with enough precision to enable accurate change detection when only a single item was presented. This result supports the conclusion that visual shortterm memory is limited by the total amount of visual information that can be stored in memory and that this capacity can be flexibly allocated to objects depending on their complexity (see Palmer, 1990, for a similar trade-off between the number of objects stored in memory and the precision with which those objects are stored).

\section{DISCUSSION}

The current experiment demonstrates that, in terms of the number of objects, visual short-term memory capacity varies across different classes of stimulus materials. Subjects can remember more of some objects, such as colored squares, than of other objects, such as Chinese characters or random polygons. The variation in the number of objects that can be stored contradicts any model of visual short-term memory that proposes that capacity is fixed solely in terms of the number of objects. Moreover, the current experiment shows an inverse relation between the information load per object and the number of objects that can be stored. This suggests that there is an upper bound on storage that is set in terms of the total amount of information. More capacity must be allocated to more complex stimuli, and consequently there is a trade-off between the complexity of the objects and the total number of objects that can be stored in memory.

Although our data show a capacity limit in terms of information, we found strong evidence that storage is indeed also limited in terms of number of objects. Specifically, the linear relation we found between visual search rate and the reciprocal of memory capacity shows how the expected capacity increases as the information per item becomes smaller and smaller. Most important, as the information per item approaches zero, the capacity grows only to a maximum of four or five items (the intersection of the linear function with the axis representing capacity). This result suggests that even when little or no individuating information is being stored for each item, only four or five items can fit in storage and that this limit cannot be exceeded for any type of stimulus. Thus, both the total information load and the number of objects impose capacity limits on visual short-term memory.

\section{Why Did Luck and Vogel (1997) Find That Number of Features Had No Effect?}

If there is an information limit on memory storage, why was performance unaffected by the number of features remembered per object in Luck and Vogel's (1997) experiment? It has long been established that the capacity of verbal short-term memory varies as a function of stimulus type (e.g., Brener, 1940; Jacobs, 1887), so it would be surprising if visual short-term memory did not behave similarly. Nevertheless, this variability does not detract from the striking result 
of Luck and Vogel. When the amount of detail required from each object increased from one feature to four features in their experiment, the capacity, as measured by change detection, remained constant.

There are at least two possible explanations for the apparent discrepancy between our result and that of Luck and Vogel (1997). First, there might be separate visual short-term memory stores for individual, basic features of an object, such as size, color, and orientation. In this case, reaching the capacity limit for one feature, say, size, would not prevent the subject from taking on more information about another feature, say, color. Consequently, the memory capacity for objects defined by conjunctions of these privileged features would remain as high as the memory capacity for the individual features alone. Luck and Vogel (1997) initially ruled out independent feature memories when they showed that two colors per object are remembered as well as a single color per object. However, recent work shows that objects with multiple values on a single dimension cannot be remembered as well as objects with a single value on that dimension (Wheeler \& Treisman, 2002). Thus, the model of independent feature memories still provides a workable explanation of Luck and Vogel's results and also a mechanism for reduced capacity for items with multiple values on single dimensions (multiple colors, multiple orientations, etc.). In this case, we must assume that the complex stimuli we used, such as cubes and random shapes, require more than one value per feature (say, two orientations), which would reduce the capacity estimate in the change detection task.

Second, we suggest that the minimal representation of object identity includes an obligatory set of core features that are always encoded regardless of the task demands. The number of features probed in a memory task can then be increased (as long as they are all in the core set) without increasing memory load, as all of the features are already in memory regardless of whether only one is probed or, say, any one of the four might be probed on each trial (as in Luck \& Vogel's, 1997, task). The features used by Luck and Vogel must all be in our hypothetical core set for their measured capacity to be independent of the number of features included in Luck and Vogel's conjunctions. For the more complex stimuli in our experiment, we assume that additional details beyond the basic set do need to be encoded and that there is then a trade-off: As the visual information required to discriminate between objects increases beyond the core set, the maximum number of objects that can be stored in memory decreases. The trade-off between complexity (when more than the base set of features is required) and the maximum number of objects stored corresponds to our linear function in Figure 2 .

\section{Relation Between Search Rate and Information Load}

Is the visual search rate appropriate for estimating the visual information load per item? In the visual search task we used, only one item, the target, is held in memory, and the display items must be encoded and compared to the memory representation of the target. The visual search rate therefore depends on the time required to encode and compare items. It is important to consider why this measure would reflect the information load for items stored in visual short-term memory. The encoding time and matching time would certainly indicate the amount of detail being encoded and compared, but neither of these operations is necessarily occurring in visual short-term memory. However, the target itself is in visual short-term memory, and we must assume that the display items, once encoded and stored for comparison, must be in a format comparable to the format of the memory item; otherwise, a comparison would not be possible. We feel that the encoding and comparison times for the display items are therefore a reasonable measure of the complexity of the stored representation to which they are compared.

It is important to emphasize that the assumed relation between visual search rate and the visual information load of objects does not rely critically on the actual mechanisms of visual search. Whether modeled as a serial process (Treisman \& Gelade, 1980; Wolfe, Cave, \& Franzel, 1989) or a parallel process (Eckstein, 1998; Palmer \& McLean, 1995; Townsend, 1971, 1990), the visual search rate represents the same quantity: the increase in the time required to process the display when an additional object is added. Independently of the modeling assumptions, this rate of processing reflects the additional amount of visual information that must be encoded and compared for each new item in the display, and therefore indexes the amount of visual information per object.

The advantage to using the visual search rate rather than a memory search rate or reaction times in the change detection task is that only a single object, the search target, is stored in memory. Thus, with this measure we are always evaluating memory processing while the memory load is well below its capacity. We are therefore not confounding performance measures with capacity limits.

Some readers might argue that the correlation between the searchrate and memory-capacity results is obvious and accounted for solely by difficulty. As the stimulus complexity increases, it is harder to remember the items and takes longer to process them. Indeed, this is exactly our point, but the link is not a "difficulty" factor, it is the visual information load per item, measured in two different ways in the two tasks: total number of items that can be stored with no time constraints (memory capacity) and time to process each item with no capacity constraint (visual search). The capacity measure was based on an error score estimated without time pressure, so this limit cannot be attributed to an underlying temporal processing constraint (we checked this with our variable-exposure-duration control). Moreover, the visual search rate we used to estimate complexity or visual information load per item was taken with no capacity bottleneck; only one item, the target, was held in short-term memory. Search rate, therefore, was not a trivial consequence of memory capacity influencing the search task. The two independent measures correlate, we claim, because both are determined by the visual information load per item, totaled over all memory items in the change detection task, but acting individually for the single target item in the search task. A similar inverse relation between memory search rates and memory span (Cavanagh, 1972; Puckett \& Kausler, 1984) for mostly verbal materials has been used to argue that short-term memory capacity is limited by the total information load.

Finally, although our results show that the capacity of visual shortterm memory is ultimately limited to four or five objects, as suggested by Luck and Vogel (1997), the results also show that this is not the only limit. The upper storage limit of four or five items is attainable only by the very simplest objects; as the visual information load per item increases, the storage limit drops to substantially lower levels. Thus, the capacity of visual short-term memory is defined by two 
limits: First, the total amount of visual information (the product of the number of objects and the visual information per object) cannot exceed the maximum visual information limit; second, the number of objects cannot exceed four or five.

\section{REFERENCES}

Baddeley, A. (1992). Working memory. Science, 255, 556-559.

Baddeley, A.D. (1978). The trouble with levels: A reexamination of Craik and Lockhart's framework for memory research. Psychological Review, 85, 139-152.

Brener, R. (1940). An experimental investigation of memory span. Journal of Experimental Psychology, 26, 467-482.

Cavanagh, J.P. (1972). Relation between the immediate memory span and the memory search rate. Psychological Review, 79, 525-530.

Comtois, R. (2003). Vision Shell PPC [Software libraries]. Cambridge, MA: Author.

Eckstein, M.P. (1998). The lower visual search efficiency for conjunctions is due to noise and not serial attentional processing. Psychological Science, $2,111-118$.

Jacobs, J. (1887). Experiments on "prehension." Mind, 12, 75-79.

Luck, S.J., \& Vogel, E.K. (1997). The capacity of visual working memory for features and conjunctions. Nature, 390, 279-281.

Palmer, J. (1990). Attentional limits on the perception and memory of visual information. Journal of Experimental Psychology: Human Perception and Performance, 16, 332-350.

Palmer, J., \& McLean, J. (1995, August). Imperfect, unlimited capacity, parallel search yields large set size effects. Paper presented at the annual meeting of the Society for Mathematical Psychology, Irvine, CA.
Pashler, H. (1988). Familiarity and visual change detection. Perception \& Psychophysics, 44, 369-378.

Phillips, W.A. (1974). On the distinction between sensory storage and shortterm visual memory. Perception \& Psychophysics, 16, 283-290.

Puckett, J.M., \& Kausler, D.H. (1984). Individual differences and models of memory span: A role for memory search rate? Journal of Experimental Psychology: Learning, Memory, and Cognition, 10, 72-82.

Shannon, C.E. (1948). A mathematical theory of communication. Bell System Technical Journal, 27, 379-423.

Snodgrass, J.G., \& Vanderwart, M. (1980). A standardized set of 260 pictures: Norms for name agreement, familiarity and visual complexity. Journal of Experimental Psychology: Human Learning and Memory, 6, 174-215.

Townsend, J.T. (1971). A note on the identification of parallel and serial processes. Perception \& Psychophysics, 10, 161-163.

Townsend, J.T. (1990). Serial and parallel processing: Sometimes they look like Tweedledum and Tweedledee but they can (and should) be distinguished. Psychological Science, 1, 46-54.

Treisman, A.M., \& Gelade, G. (1980). A feature-integration theory of attention. Cognitive Psychology, 12, 97-136.

Vogel, E.K., Woodman, G.F., \& Luck, S.J. (2001). Storage of features, conjunctions, and objects in visual working memory. Journal of Experimental Psychology: Human Perception and Performance, 27, 92-114.

Wheeler, M.E., \& Treisman, A.M. (2002). Binding in short-term visual memory. Journal of Experimental Psychology: General, 131, 48-64.

Wolfe, J.M., Cave, K.R., \& Franzel, S.L. (1989). Guided search: An alternative to the feature integration model for visual search. Journal of Experimental Psychology: Human Perception and Performance, 15, 419-433.

(RECEIVED 3/28/02; REvision ACCEPTED 3/14/03) 